\title{
THE DILATATION INVARIANT IN THE HOMOTOPY OF SPHERES
}

\author{
SENLIN XU and JINCHI LV
}

Received 15 May 2002

\begin{abstract}
We obtain some results on the filtration invariant in the homotopy of spheres. Then by the relation between the dilatation invariant and filtration invariant, we get the corresponding results on the dilatation invariant in the homotopy of spheres.
\end{abstract}

2000 Mathematics Subject Classification: 55Q40, 57R35.

1. Introduction. Let $S^{m}$, respectively $S^{n}$, denote the standard unit sphere in Euclidean space $\mathbb{R}^{m+1}$, respectively $\mathbb{R}^{n+1}$, and let $f: S^{m} \rightarrow S^{n}$ be a smooth map. Following Olivier [5], we define $\delta(f)$, the dilatation of $f$, by

$$
\delta(f)=\sup _{|X|=1}|d f(X)|
$$

where $X$ ranges over all unit tangent vectors and $|\cdot|$ is a Euclidean metric. Moreover, if $\alpha \in \pi_{m}\left(S^{n}\right)$, we define $\delta(\alpha)$, the dilatation invariant of $\alpha$, to be the real number

$$
\delta(\alpha)=\inf _{f} \delta(f)
$$

where $f: S^{m} \rightarrow S^{n}$ ranges all smooth representatives $f$ of $\alpha$. It has been conjectured by Olivier [5] that $\delta(\alpha)$ is always an integer, which is still an open problem.

The filtration invariant of $\alpha \in \pi_{m}\left(S^{n}\right)$, filt $(\alpha)$, is the integer originally defined by James [2] in connection with his study of the reduced product construction. The definition is as follows [6]. For $p, q \in S^{n}, n \geq 2$, let $\Omega^{*}=$ $\Omega^{*}\left(S^{n} ; p, q\right)$ be the space of all continuous paths $\omega:[0,1] \rightarrow S^{n}$ with $\omega(0)=p$, $\omega(1)=q$, equipped with the compact-open topology. As well known, if $p$ and $q$ are not conjugate on $S^{n}$, that is, $q \neq p, p^{\prime}$ where $p^{\prime}$ denotes the antipode of $p$, then $\Omega^{*}$ admits a $C W$-decomposition of the form

$$
\Omega^{*} \simeq S^{n-1} \cup e^{2(n-1)} \cup \cdots \cup e^{r(n-1)} \cup \cdots .
$$

Let $\Omega_{r(n-1)}^{*} \simeq S^{n-1} \cup e^{2(n-1)} \cup \cdots \cup e^{r(n-1)}$ be its $r(n-1)$-skeleton. This allows us to define a filtration on the homotopy group $\pi_{m}\left(S^{n}\right)$ as follows. For any 
$\alpha \in \pi_{m}\left(S^{n}\right)$, filt $(\alpha)$ is the least integer $r \geq 0$ for which the adjoint of $\alpha$, ad $(\alpha) \in$ $\pi_{m-1}\left(\Omega^{*}\right)$, lies in the image of the naturally induced map

$$
\pi_{m-1}\left(\Omega_{r(n-1)}^{*}\right) \longrightarrow \pi_{m-1}\left(\Omega^{*}\right)
$$

One important fact is that filt $(\alpha)=0$ if and only if $\alpha=0$ and filt $(\alpha) \leq 1$ if and only if $\alpha$ is a suspension element.

The composition problem is very interesting. As we all know, filt $(\cdot)$ and $\delta(\cdot)$ are "submultiplicative," that is,

$$
\text { filt }(\alpha \circ \beta) \leq \text { filt }(\alpha) \cdot \text { filt }(\beta), \quad \delta(\alpha \circ \beta) \leq \delta(\alpha) \cdot \delta(\beta)
$$

The former is verified in [2] and the latter is obvious. If $\alpha \in \pi_{2 n-1}\left(S^{n}\right)$ is an element of Hopf invariant unity, then we have filt $(\alpha \circ \beta)=2$ filt $(\beta)=\operatorname{filt}(\alpha)$. filt $(\beta)$ from [2]. With the same assumption, it has been conjectured by Roitberg [6] that $\delta(\alpha \circ \beta)=\delta(\alpha) \cdot \delta(\beta)$. But, unfortunately, this conjecture is not true even for the Hopf homotopy class.

In [6], Roitberg got a relation between the dilatation and filtration invariants in the homotopy of spheres by the fundamental theorem of Morse theory. In this note, we want to exploit more of the dilatation invariant. First, we get the following theorem, which is a generalization of [7, Theorem 1].

THEOREM 1.1. Let $m>n \geq 2, f: S^{m} \rightarrow S^{n}$ be a smooth map and $\lambda=$ $\max _{x \in S^{n}} d(f(x), f(-x))$, where $d$ is the usual unit sphere metric. If filt $([f])=$ $r$, then $\delta(f) \geq 2[(r+1) / 2]+\left(1+(-1)^{r}\right) \lambda / 2 \pi$.

2. Proof of Theorem 1.1. Let $\Omega=\Omega\left(S^{n} ; p, q\right)$ be the space of all piecewise smooth paths $\omega:[0,1] \rightarrow S^{n}$ with $\omega(0)=p$, $\omega(1)=q$, equipped with the topology induced by the metric $d^{*}$ defined in Milnor [4], let $E: \Omega \rightarrow \mathbb{R}$ be the energy function defined by $E(\omega)=\int_{0}^{1}|d \omega / d t|^{2} d t$, and set

$$
\Omega^{c}=\Omega^{c}\left(S^{n} ; p, q\right)=E^{-1}\left(\left[0, c^{2}\right]\right), \quad c \geq 0 .
$$

It is well known [4, Theorem 17.1] that the inclusion $i: \Omega \rightarrow \Omega^{*}$ is a homotopy equivalence. Naturally, $f$ induces a map

$$
\tilde{f}: S^{m-1}=\Omega^{\pi}\left(S^{m} ; x,-x\right) \longrightarrow \Omega^{\delta(f) \pi}=\Omega^{\delta(f) \pi}\left(S^{n} ; f(x), f(-x)\right)
$$

for any $x \in S^{m}$, and the composition

$$
i \circ j \circ \tilde{f}: S^{m-1} \longrightarrow \Omega^{*},
$$

$j: \Omega^{\delta(f) \pi} \rightarrow \Omega$ the inclusion, is precisely the adjoint of $f$. The proof then follows from a study of the homotopy type of $\Omega^{\delta(f) \pi}$ for suitable choice of $x \in S^{m}$. 
The Borsuk-Ulam theorem guarantees the existence of a point $x \in S^{m}$ such that $f(x)=f(-x)$. So, $\min _{x \in S^{m}} d(f(x), f(-x))=0$. We first assume that $\lambda>$ 0 . Suppose that $\delta(f)<2[(r+1) / 2]+(-1)^{r} \lambda / \pi$. Let $\mu$ be a number such that $0<\mu<\lambda \leq \pi$ and

$$
\delta(f)<2\left[\frac{r+1}{2}\right]+(-1)^{r} \mu / \pi .
$$

Thus, by the continuity of $d$, there is a point $x \in S^{m}$ such that

$$
d(f(x), f(-x))=\mu .
$$

From (2.4), we have $\delta(f) \pi<2[(r+1) / 2] \pi+(-1)^{r} \mu$. Hence, by (2.5) and the fundamental theorem of Morse theory [4, Theorem 17.3 and Corollary 17.4], we deduce the following commutative diagram

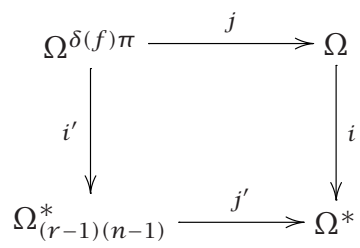

where $j^{\prime}$ is the inclusion and $i^{\prime}$ is a homotopy equivalence. The conclusion filt $([f]) \leq r-1$ then follows from (2.3), which is a contradiction. Furthermore, when $r$ is odd, we can get $\delta(f) \geq r+1$ by letting $\mu \rightarrow 0^{+}$. Then, we consider the case that $\lambda=0$. For any $\epsilon>0$, we can choose a suitable $C^{1}$-approximation $g$ of $f$ such that $[g]=[f],|\delta(g)-\delta(f)|<\epsilon$, and $\lambda(g)<\epsilon$. Letting $\epsilon \rightarrow 0^{+}$, we can get the conclusions.

REMARK 2.1. Noting that if filt $(\alpha)=r$ is even and $\inf _{f} \lambda(f)=\mu>0$, then we have $\delta(\alpha) \geq r+\mu / \pi$. If $\delta(\alpha)$ is an integer, then we could get $\delta(\alpha) \geq r+1$. The case that $\mu=\pi$ is very interesting since if true we can get $\delta(\alpha) \geq r+1$. When $m$ is even and $2 \alpha \neq 0$, Lawson Jr. [3] showed that for any representative $f$ of $\alpha$, there exists a pair of antipodal points mapped by $f$ into an antipodal pair, which had been contained in [7, Theorem 1].

COROLLARY 2.2. If $\alpha \in \pi_{m}\left(S^{2}\right)$ with $m>2$ and $2 \alpha \neq 0$, then $\delta(\alpha) \geq 4$.

Proof. According to [1], filt $(\alpha)>3$; so filt $(\alpha)=r \geq 4$. Applying Theorem 1.1 , we have

$$
\delta(f) \geq 2\left[\frac{r+1}{2}\right]+\frac{\left(1+(-1)^{r}\right) \lambda}{2 \pi} \geq 4
$$

for any smooth representative $f$ of $\alpha$. Thus, $\delta(\alpha) \geq 4$.

3. The case of $S^{3}$. In this section, we consider the filtration of any element in $\pi_{3+k}\left(S^{3} ; p\right)$ for $k=2 p-3,2(i+1)(p-1)-2$, and $2(i+1)(p-1)-1$, 
$i=1, \ldots, p-1$, where $p$ is an odd prime and $\pi_{m}\left(S^{n} ; p\right)$ represents the $p$ primary component of the Abelian group $\pi_{m}\left(S^{n}\right)$. From [8, Theorem 13.4], we have the following two propositions.

Proposition 3.1. Let $p$ be an odd prime, then $\pi_{3+k}\left(S^{3} ; p\right)$ is $\mathbb{Z}_{p}$ for $k=$ $2 p-3,2(i+1)(p-1)-2$, and $2(i+1)(p-1)-1, i=1, \ldots, p-1$, and is 0 otherwise for $k<2(p+1)(p-1)-3$.

Proposition 3.2. Let $p$ be an odd prime, then $\pi_{2 m p-1+k}\left(S^{2 m p-1} ; p\right)$ is $\mathbb{Z}_{p}$ for $k=2 i(p-1)-1,1 \leq i<p, m \geq 1$, and is 0 otherwise for $k<2 p(p-1)-2$, $m \geq 1$.

We identify $S^{m}$ with the suspension of $S^{m-1}$ in the usual way. Then, we have an isomorphism $\phi: \pi_{m}\left(S^{n}\right) \rightarrow \pi_{m-1}\left(S_{\infty}^{n-1}\right)$ (see [2]). For any element $\alpha$ in $\pi_{m}\left(S^{n}\right)$ of order $q$ which is not necessarily a prime, $\phi(\alpha)$ is also an element of order $q$ in $\pi_{m-1}\left(S_{\infty}^{n-1}\right)$. Let filt $(\alpha)=r$, then $\phi(\alpha)$ lies in the image of the natural homomorphism $\pi_{m-1}\left(S_{r}^{n-1}\right) \rightarrow \pi_{m-1}\left(S_{\infty}^{n-1}\right)$. When we consider the natural homomorphisms

$$
\pi_{m-1}\left(S_{r}^{n-1}\right) \longrightarrow \pi_{m}\left(S_{k}^{n-1}\right) \longrightarrow \pi_{m-1}\left(S_{\infty}^{n-1}\right),
$$

we know that $\phi(\alpha)$ is also an element of order $q$ in $\pi_{m-1}\left(S_{k}^{n-1}\right)$ for $k \geq r$. So, we have the following proposition.

Proposition 3.3. If $\pi_{m-1}\left(S_{k}^{n-1} ; p\right)=0$, then filt $(\alpha) \geq k+1$ for any nonzero element $\alpha \in \pi_{m}\left(S^{n} ; p\right)$.

As we all know, $\pi_{n}\left(S^{n}\right) \cong \mathbb{Z}$, so $\pi_{2+k}\left(S^{2 p-1} ; p\right)=0$ for $k=2 p-3$. By Proposition 3.2, we have

$$
\pi_{2+k}\left(S^{2 p-1} ; p\right)=0 \quad \text { for } k=2(i+1)(p-1)-1, i=1, \ldots, p-1 \text {. }
$$

Thus, by the isomorphism $\pi_{i}\left(S_{p-1}^{2} ; p\right) \rightarrow \pi_{i}\left(S^{2 p-1} ; p\right)$ for $i \geq 2$ and Proposition 3.3 , we get the following theorem.

THEOREM 3.4. The elements of order $p$ in $\pi_{3+k}\left(S^{3}\right)$ are of filtration at least $p$ for $k=2 p-3$ and $2(i+1)(p-1)-1, i=1, \ldots, p-1$.

By Theorems 1.1 and 3.4, we can easily get the following corollary.

COROLLARY 3.5. The elements of order $p$ in $\pi_{3+k}\left(S^{3}\right)$ are of dilatation at least $p+1$ for $k=2 p-3$ and $2(i+1)(p-1)-1, i=1, \ldots, p-1$.

COROLLARY 3.6. If $\alpha$ is an element of order $p$ in $\pi_{3+k}\left(S^{3}\right)$ as in Corollary 3.5 and $\gamma \in \pi_{3}\left(S^{2}\right)$ is the Hopf class, then $\delta(\gamma \circ \alpha) \geq 2 p+1$.

Proof. According to [2], filt $(\gamma \circ \alpha)=2$ filt $(\alpha)$ since $\gamma$ has Hopf invariant one. Noting that the composition is a homomorphism, the order of $\gamma \circ \alpha$ is 
then 1 or $p$ as $p$ is prime. As filt $(\alpha) \geq p$ by Theorem 3.4, we have filt( $\gamma \circ$ $\alpha)=2$ filt $(\alpha) \geq 2 p>0$, so $\gamma \circ \alpha \neq 0$. Thus, the order of $\gamma \circ \alpha$ is $p$. It follows immediately from Remark 2.1 that $\delta(\gamma \circ \alpha) \geq 2 p+1$ since $3+k$ is even and $p$ is an odd prime.

From [8, Theorem 13.4], we know that $\pi_{2 p-3+k}\left(S^{2 p-3} ; p\right)$ is $\mathbb{Z}_{p}$ for $k=2(p-$ $1)^{2}-2$ and $2 i(p-1)-1, i=1, \ldots, p-1$, and is 0 otherwise for $k<2 p(p-1)-2$. So we have

$$
\pi_{2+k}\left(S^{2 p-3} ; p\right)=0 \text { for } k=2(i+1)(p-1)-2, i=1, \ldots, p-1 .
$$

Thus, by the isomorphism $\pi_{i}\left(S_{p-2}^{2} ; p\right) \rightarrow \pi_{i}\left(S^{2 p-3} ; p\right)$ and Proposition 3.3, we get the following proposition.

Proposition 3.7. The elements of order $p$ in $\pi_{3+k}\left(S^{3}\right)$ are of filtration at least $p-1$ for $k=2(i+1)(p-1)-2, i=1, \ldots, p-1$.

By Theorem 1.1 and Proposition 3.7, we can easily get the following corollary.

COROLLARY 3.8. The elements of order $p$ in $\pi_{3+k}\left(S^{3}\right)$ are of dilatation at least $p-1$ for $k=2(i+1)(p-1)-2, i=1, \ldots, p-1$.

COROLlaRY 3.9. If $\alpha$ is an element of order $p$ in $\pi_{3+k}\left(S^{3}\right)$ as in Corollary 3.8 and $\gamma \in \pi_{3}\left(S^{2}\right)$ is the Hopf class, then $\delta(\gamma \circ \alpha) \geq 2(p-1)$.

Proof. According to [2], filt $(\gamma \circ \alpha)=2$ filt $(\alpha)$ since $\gamma$ has Hopf invariant one. As filt $(\alpha) \geq p-1$ by Proposition 3.7, it follows immediately from Theorem 1.1 that $\delta(\gamma \circ \alpha) \geq 2(p-1)$.

4. Further results on the case that $\pi_{m}\left(S^{n} ; p\right)=\mathbb{Z}_{p}$. Let $p$ be a prime. We consider the case that $\pi_{m}\left(S^{n} ; p\right)=\mathbb{Z}_{p}$. For any element $\alpha$ of order $p$ in $\pi_{m}\left(S^{n}\right)$, we denote by $\lambda(\alpha)$ the least integer $k$ such that $\pi_{m-1}\left(S_{k}^{n-1} ; p\right) \neq 0$, then we have

$$
\pi_{m-1}\left(S_{\lambda(\alpha)-1}^{n-1} ; p\right)=0, \quad \pi_{m-1}\left(S_{\lambda(\alpha)}^{n-1} ; p\right)=\mathbb{Z}_{p}
$$

Thus by Proposition 3.3, we have

$$
\text { filt }(\alpha) \geq \lambda(\alpha) \text {. }
$$

Let $\beta$ be an element of order $p$ in $\pi_{m-1}\left(S_{\lambda(\alpha)}^{n-1}\right)$, then $\beta$ can be naturally regarded as an element in $\pi_{m-1}\left(S_{\infty}^{n-1}\right)$ with order $p$. Then, $\phi^{-1}(\beta)$ is an element of order $p$ in $\pi_{m}\left(S^{n}\right)$. Since $\pi_{m}\left(S^{n} ; p\right)=\mathbb{Z}_{p}$, we have

$$
\pi_{m}\left(S^{n} ; p\right)=\left\langle\phi^{-1}(\beta)\right\rangle
$$


So, $\alpha=k \phi^{-1}(\beta)$ for some $1 \leq k \leq p-1$. Thus, $\alpha=k \phi^{-1}(\beta)=\phi^{-1}(k \beta)$ and $\phi(\alpha)=k \beta \in \pi_{m-1}\left(S_{\lambda(\alpha)}^{n-1}\right)$. So we have

$$
\text { filt }(\alpha) \leq \lambda(\alpha)
$$

By (4.2) and (4.4), we get the following theorem.

THEOREM 4.1. If $\pi_{m}\left(S^{n} ; p\right)=\mathbb{Z}_{p}$, then filt $(\alpha)=\lambda(\alpha)$ for any element $\alpha$ of order $p$ in $\pi_{m}\left(S^{n}\right)$.

By Proposition 3.2, we have

$$
\pi_{2+k}\left(S^{2 p-1} ; p\right)=\mathbb{Z}_{p} \quad \text { for } k=2(i+1)(p-1)-2, i=1, \ldots, p-1 .
$$

So we have $\lambda(\alpha)=p-1$ for any element $\alpha$ of order $p$ in $\pi_{3+k}\left(S^{3}\right)$ where $k=2(i+1)(p-1)-2, i=1, \ldots, p-1$. Thus by Theorem 4.1 , we get the following theorem.

THEOREM 4.2. The elements of order $p$ in $\pi_{3+k}\left(S^{3}\right)$ are of filtration $p-1$ for $k=2(i+1)(p-1)-2, i=1, \ldots, p-1$.

\section{REFERENCES}

[1] I. M. James, The suspension triad of a sphere, Ann. of Math. (2) 63 (1956), 407-429.

[2] - Filtration of the homotopy groups of spheres, Quart. J. Math. Oxford Ser. (2) 9 (1958), 301-309.

[3] H. B. Lawson Jr., Stretching phenomena in mappings of spheres, Proc. Amer. Math. Soc. 19 (1968), 433-435.

[4] J. W. Milnor, Morse Theory, Annals of Mathematics Studies, no. 51, Princeton University Press, New Jersey, 1963.

[5] R. Olivier, Über die Dehnung von Sphärenbbildungen, Invent. Math. 1 (1966), 380390 (German).

[6] J. Roitberg, Dilatation phenomena in the homotopy groups of spheres, Advances in Math. 15 (1975), 198-206.

[7] _ The dilatation and filtration invariants in the homotopy of spheres, Advances in Math. 20 (1976), no. 2, 280-284.

[8] H. Toda, Composition Methods in Homotopy Groups of Spheres, Annals of Mathematics Studies, no. 49, Princeton University Press, New Jersey, 1962.

Senlin Xu: Department of Mathematics, The University of Science and Technology of China, Hefei 230026, China

Jinchi Lv: Department of Mathematics, The University of Science and Technology of China, Hefei 230026, China

E-mail address: 1vxi@mai 1 . ustc. edu.cn 


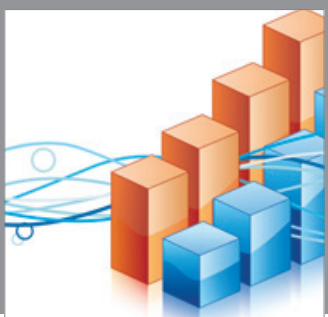

Advances in

Operations Research

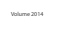

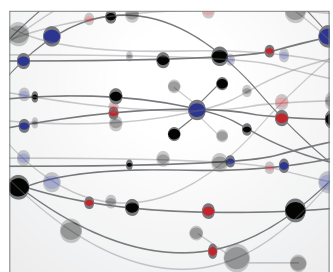

\section{The Scientific} World Journal
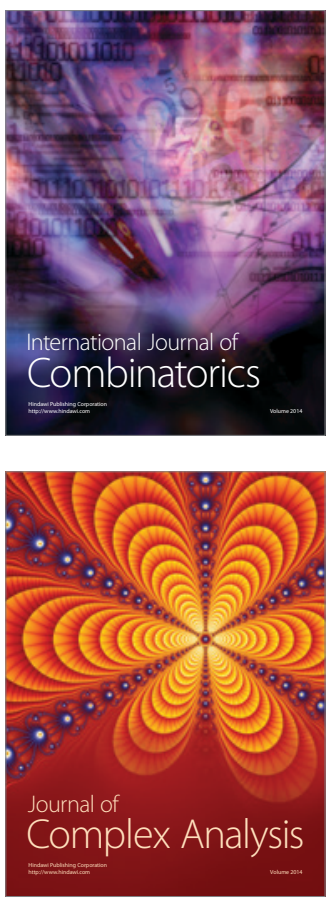

International Journal of

Mathematics and

Mathematical

Sciences
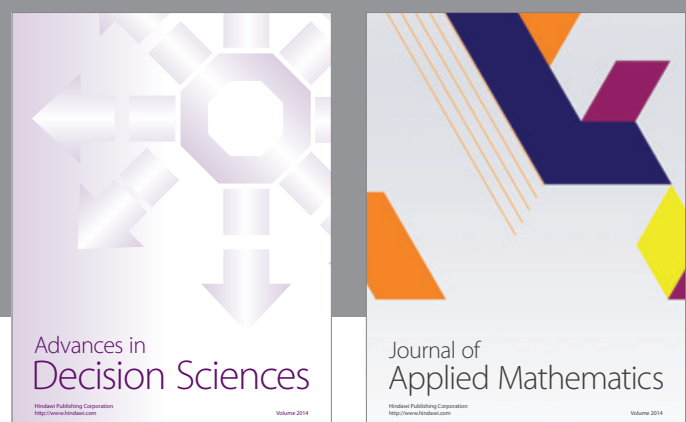

Journal of

Applied Mathematics
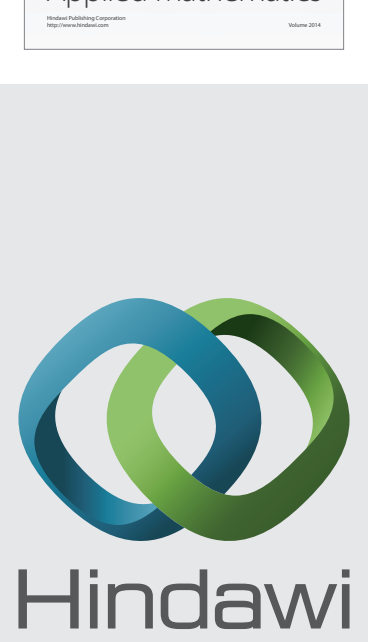

Submit your manuscripts at http://www.hindawi.com
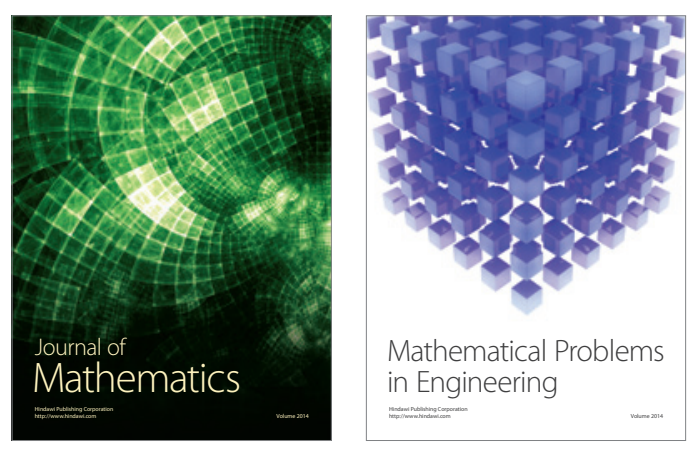

Mathematical Problems in Engineering
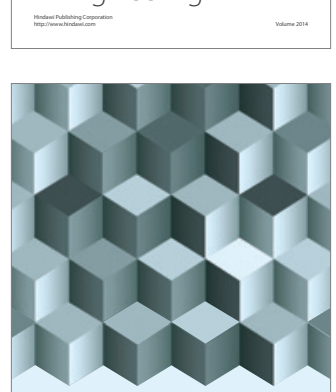

Journal of

Function Spaces
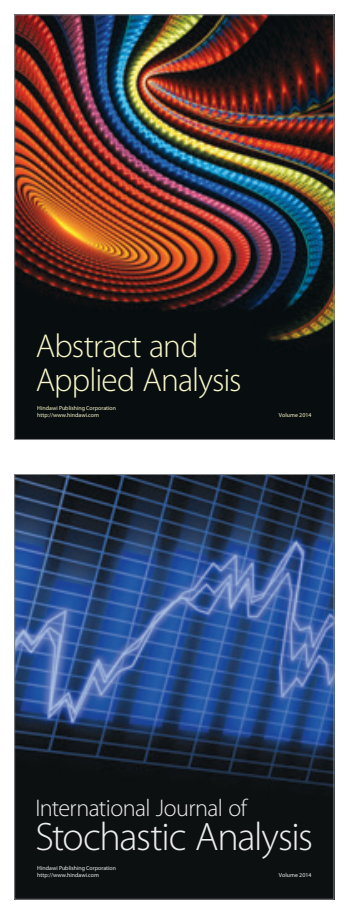

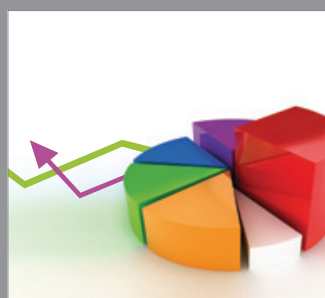

ournal of

Probability and Statistics

Promensencen
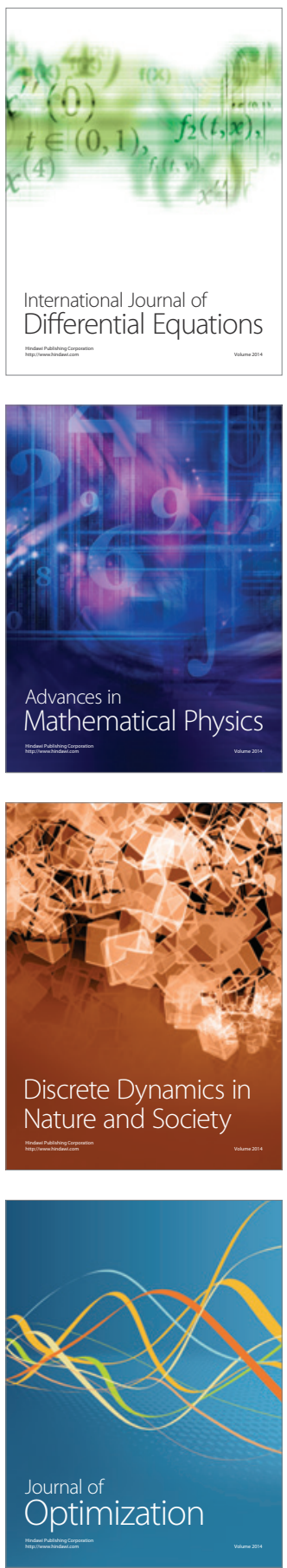